\title{
The field of the equatorial electrojet from CHAMP data
}

\author{
J.-L. Le Mouël ${ }^{1}$, P. Shebalin ${ }^{2}$, and A. Chulliat ${ }^{1}$ \\ ${ }^{1}$ Laboratoire de Géomagnétisme, Institut de Physique du Globe de Paris, 4 place Jussieu 75252 Paris cedex 05, France \\ ${ }^{2}$ International Institute of Earthquake Prediction Theory and Mathematical Geophysics, Moscow 113556, Russia
}

Received: 23 June 2005 - Revised: 25 November 2005 - Accepted: 3 January 2006 - Published: 23 March 2006

\begin{abstract}
We apply a simple linear transform, the alongtrack second derivative, to four years of scalar and vectorial data from the CHAMP satellite. This transform, reminiscent of techniques used in the interpretation of aeromagnetic surveys, is applied either to the geocentric spherical components of the field or to its intensity. After averaging in time and space, we first produce a map of the crustal field, then maps of the equatorial electrojet field at all local times and all universal times. The seasonal variation of the electrojet, its evolution with the solar cycle, and the effect of geomagnetic activity are discussed. The variation of the electrojet with longitude, an intriguing feature revealed by satellite data, is described in some detail, and it is shown that this longitude dependance is stable in time. The existence of a counterelectrojet in the morning, everywhere except over the Pacific Ocean, is established. The signatures of closure electric currents and of interhemispheric currents are also evidenced.
\end{abstract}

Keywords. Geomagnetism and paleomagnetism (Time variations, diurnal to secular) - Ionosphere (Electric fields and currents; Equatorial ionosphere)

\section{Introduction}

The equatorial electrojet was discovered after the establishment of a geomagnetic observatory at Huancayo, near the dip equator. It appeared as an abnormally large amplitude of daily variation in the equatorial component $H$. This enhancement was attributed by Egedal (1947) to a band of electric current about $300 \mathrm{~km}$ wide, flowing along the geomagnetic dip equator in the ionospheric $\mathrm{E}$ region. It was named the equatorial electrojet (EEJ hereafter) by Chapman in 1951 (see Rastogi, 1989, for a history of the discovery of the electrojet).

The EEJ is due to a local enhancement of the ionospheric conductivity in the direction parallel to the geomagnetic dip

Correspondence to: A. Chulliat

(chulliat@ipgp.jussieu.fr) equator. This effect, known as the Cowling effect, is caused by the establishment of a strong, vertical polarisation electric field in the equatorial region, where the magnetic field lines are nearly horizontal. The EEJ flows eastward, like the $S_{q}$ currents at low geomagnetic latitudes. A large number of studies have been devoted to the ionosphere in the equatorial region (see Forbes, 1981; Rastogi, 1989, for reviews).

Up until recently, most results about the magnetic field produced by the EEJ came from ground data. These data were acquired in equatorial observatories and by chains of magnetometers installed along the north-south profiles across the dip equator in South America (Forbush and Casaverde, 1961; Rigoti et al., 1999), Africa (Fambitakoye and Mayaud, 1976; Doumouya et al., 1998) and India (Rastogi, 1989). Unfortunately, no truly global picture of the EEJ can be inferred from ground data only, since a large fraction of the dip equator lies over the ocean. The Magsat satellite only partially improved the situation as it provided a global picture of the EEJ field only around 06:00 and 18:00 local times, where the EEJ is very weak and cannot easily be separated from the underlying crustal field (Cohen, 1989; Cohen and Achache, 1990; Langel et al., 1993; Sabaka et al., 2002).

The situation has very much improved since the launch of the Ørsted and CHAMP satellites in 1999 and 2000, respectively. Both low-Earth orbiting satellites have been providing continuous global coverage of the magnetic field with unprecedented precision, at all local times. Relying on this new data set, several studies have been devoted to the global structure of the EEJ and its variability with season and geomagnetic activity, either using Ørsted data (Jadhav et al., 2002a,b; Ivers et al., 2003) or CHAMP data (Lühr et al., 2004). An outstanding issue in all these studies is the variation with longitude of the EEJ maximum intensity. So far no agreement on the shape of longitude profile has been reached and the origin of this variation is left unexplained.

The goal of the present paper is to rely on CHAMP data to produce global maps of the magnetic field anomaly generated by the EEJ, at all local times and all universal times, and to investigate how this anomaly changes with longitude,

Published by Copernicus GmbH on behalf of the European Geosciences Union. 
season and geomagnetic activity. CHAMP data are actually more suited to EEJ studies because CHAMP flies at a lower altitude (about $400 \mathrm{~km}$ instead of $750 \mathrm{~km}$ ), i.e. closer to the EEJ, and undergoes a quicker drift in local time. We will keep here to a descriptive point of view, not resorting to any kind of modeling, except for the main field, and using instead a method somewhat inspired by geophysical prospecting.

\section{Data and analysis}

In the present paper, we use CHAMP one-second data acquired during the time span 2001-2004. The CHAMP satellite, launched in 2000 , is ideally suited for studying the equatorial electrojet. Orbiting at a slowly decreasing altitude between 350 and $450 \mathrm{~km}$, it has been providing nearEarth, high-precision magnetic data at all local times for about 4 years. The three components of the field are measured using a fluxgate tri-axial sensor and the intensity of the field is measured using an Overhauser magnetometer. Data are acquired every one second. We consider all data provided by both instruments over 2001-2004, without omitting data points based on geomagnetic selection criteria (except as in Sect. 3.6).

Our goal is to separate the EEJ field from the other components of the Earth's magnetic field: the main field, the crustal field and the part of the external field produced by other sources than the EEJ. We proceed in the following manner.

\subsection{Computing the along-track derivative}

The data used in this study are sampled every one second; the distance $\Delta l$ covered by the satellite in one second is about $7 \mathrm{~km}$. We place a data point $P$ on the orbit through its time measurement $t$ (in seconds; $t$ is an integer). We estimate the first and second along track derivatives of the $X$ component at point $P$ using the formulae:

$\dot{X}_{k}(t)=\frac{X(t+k)-X(t)}{k}$

and

$\ddot{X}_{k}(t)=\frac{X(t+k)-2 X(t)+X(t-k)}{k^{2}}$,

where $\mathrm{k}$ is also expressed in seconds. Formulae (1) and (2) are applied at each data point $P(t)$, so no information is lost. In fact, in this paper we will use only the second derivative (2). It is well known and obvious that the value of the operator (2) greatly varies with the wavelength of the signal $X(t)$, or $X(t \Delta l)$.

\subsubsection{Removing the main field}

It is not essential in the following analysis to remove the main field, by which we mean the core field represented by the spherical harmonics expansion model, but we have done so for reasons mentioned later. This field is large compared to the EEJ field at the satellite and ground altitudes, but it is broader in scale. The typical length scale of an EEJ profile perpendicular to the dip equator is $500 \mathrm{~km}$ (Rastogi, 1989), while the length scale of the $\mathrm{n}$-th degree harmonics of the spherical harmonic expansion is $40000 / n \mathrm{~km}$. Looking at the well known "spectrum" of the main field, extending from $n=1$ to $n=13$ (it is generally believed that the core field dominates the internal field up to degrees 13 or 14, whereas the crustal field dominates for higher degrees), it appears that the second derivative operator leaves behind a small contribution of the main field. Furthermore, we will consider day-night differences which will further reduce this contribution.

However, in what follows the data points $P$ will be distributed into bins whose altitude will be assumed uniform. The real distribution of altitudes is complex, and even when considering a large number of points $P$ distributed over a full year, the mean altitude may vary from bin to bin. In addition, as the main field is large, a significant noise may result. For this reason, a degree 13 main field model at epoch 2000 computed by Langlais et al. (2003) was substracted from the data. The result is denoted $\Delta \mathbf{B}=\mathbf{B}-\mathbf{B}_{\text {model }}$ for the vector field, $\Delta X, \Delta Y, \Delta Z$ for each component and $\Delta F$ for the field intensity.

\subsubsection{The choice of the step $k$}

Let us consider an along-track profile $X(t)$ (or $X(t \Delta l), t=1$, $2 \ldots$ in s). To simplify the writing, we will still write $X, Y$ and $Z$ for the components of $\Delta \mathbf{B}$ in the present paragraph. In the absence of noise, we could take $k=1$ in Eq. (2), in order to obtain the most accurate local estimate of $\ddot{X}$. But $X$ is affected by a strong noise $\xi$ (which we will not analyse here):

$X_{m}(t)=X(t)+\xi(t)$,

where $m$ is for "measured"; let us suppose $\xi$ is uncorrelated, or poorly correlated. The second difference $\xi(t+k)+\xi(t-k)-2 \xi(t)$ does not change with $k$, whereas the second difference $X(t+k)+X(t-k)-2 X(t)$ increases with $k$; so the noise contribution to Eq. (2) decreases rapidly with $k$. But we cannot increase $k$ beyond some limit: it must remain small enough for Eq. (2) to be a good estimate of $\ddot{X}$ for the wavelengths we want to study - here the characteristic length of the EEJ field.

Let $X(t)$ be represented on the CHAMP orbit great circle of radius $R$ by

$X(t)=\sum_{m} A_{m} \exp (i m v t / R)$,

where $v \approx 7 \mathrm{~km} / \mathrm{s}$. Then the first and second derivatives of $X(t)$, using a step $k$, will have coefficients $A_{m}^{k, j}$ related to the $A_{m}$ as

$\left|A_{m}^{k, j}\right|=G_{m}^{k, j}\left|A_{m}\right|$, 
where

$G_{m}^{k, j}=\frac{1}{k^{j}}[2-2 \cos (m v k / R)]^{j / 2}$

and $j$ is the order of the derivative. $G_{m}^{k, j}$ is the gain factor for the $A_{m}^{k, j}$ coefficient (see Olsen, 2004). It is plotted as a function of the wavelength $\lambda=2 \pi R / m$ for $j=2$ and three different values of $k$ on Fig. 1. The maximum gain for $j=2$ and $k=20 \mathrm{~s}$ is 0.01 at wavelength $\lambda_{\max }=2 k v \approx 280 \mathrm{~km}$.

In the present paper we chose $k=20 \mathrm{~s}$, after checking that the results are stable for $15<k<25$. For values of $k$ between $15 \mathrm{~s}$ and $25 \mathrm{~s}$, Eq. (2) provides us with a true spatial derivative which fits our needs. The daily variation $S_{R}$ and the ring current field have far longer spatial length scales than the EEJ field and are eliminated (or at least much reduced) by the selected second derivative filter. (Moreover, part of the ring current field is included in the main field model.)

Note: It is rather easy to determine that the minimum value of $k$ for the noise contribution to Eq. (2) is smaller compared to $\ddot{X}$; to this aim, one takes the absolute value of the second difference in Eq. (2) and looks at the evolution of the results with $k$.

\subsection{Computing the longitudinal component}

Let us denote by $\mathbf{b}$ the anomaly vector after applying the along-track second derivative $(\mathbf{b}=\ddot{\Delta} \mathbf{B})$. Its components in geocentric coordinates are $\ddot{\Delta X}, \ddot{\Delta} Y$, and $\ddot{\Delta} Z$. Its projection on the direction of the main field $\mathbf{B}$, i.e.

$b_{l}=\mathbf{b} \cdot \frac{\mathbf{B}}{|\mathbf{B}|}$,

will be called the longitudinal component of the anomaly field. We have that

$b_{l}=\alpha \ddot{\Delta} X+\beta \ddot{\Delta Y}+\gamma \ddot{\Delta Z}$,

where $\alpha, \beta$ and $\gamma$ are the direction cosines of $\mathbf{B}$, i.e. the cosines of the angles between $\mathbf{B}$ and the $x, y$ and $z$ axes. The longitudinal component $b_{l}$ is more suited to the study of the EEJ than b, because it is much less affected by instrumental noise due to uncertainties in attitude determination, and by field-aligned currents (which generate transverse fields). However, Cartesian components of $\mathbf{b}$ also bring valuable information, as will be seen in the following section.

It is possible to perform a very similar analysis using scalar data only. $F$ can be $\sqrt{X^{2}+Y^{+} Z^{2}}$ or given by the scalar magnetometer. The along-track second derivative of the field intensity may be expressed as

$\ddot{\Delta F}=\alpha \ddot{\Delta X}+\beta \ddot{\Delta} Y+\gamma \ddot{\Delta} Z+\dot{\alpha} \Delta \dot{X}+\dot{\beta} \Delta \dot{Y}+\dot{\gamma} \dot{\Delta} Z$.

Due to the long wavelengths of $\alpha, \beta, \gamma$ and the short wavelengths of the EEJ field, the last three terms of the RHS of (9) are much smaller than the first three terms and therefore we have that

$\stackrel{\Delta F}{\Delta F} b_{l}$.

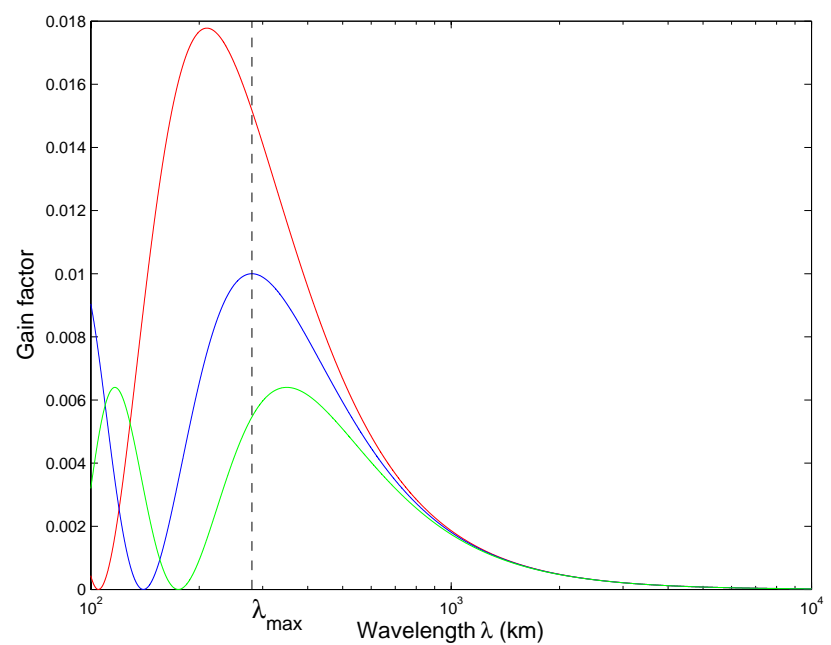

Fig. 1. Gain factor of the along-track second derivative as a function of the wavelength of the signal, using a step $k=15 \mathrm{~s}$ (red curve), $k=20 \mathrm{~s}$ (blue curve) and $k=25 \mathrm{~s}$ (green curve). The wavelength $\lambda_{\text {max }} \approx 280 \mathrm{~km}$ associated with the maximum gain for $k=20 \mathrm{~s}$ is indicated on the graph.

Using $X, Y, Z$ and $F$ data, we have checked that there are only very minor differences between maps computed from $\ddot{\Delta F}$ and maps computed from $b_{l}$. In what follows we will show results for the longitudinal component (7) only, but all maps have also been computed using the scalar second derivative (9).

\subsection{Averaging in space and time}

We average both in time and space, trying to lose as little information as possible. Time averaging, over time spans much longer than one day, is used to smooth the big day-today variability of the electrojet. It is also necessary to consider long time spans in order to have enough individual measurements to average both in time and space. Instrumental noise and instantaneous fields of various origins are always present. Although we usually consider all the data acquired from 2001 to 2004, we sometimes use them after creating different subsets (for example, 2001, 2002, 2003 and 2004), in order to investigate the stability of different patterns of their temporal variations.

For a given subset, we distribute the data within bins of size $2.5^{\circ} \times 2.5^{\circ}$ in latitude and longitude, without sorting the altitudes. The total number of bins is 10368 . For each bin $i$, we compute the average of $\ddot{X}(P)$ over all points $P$ within the bin; the same is done for $Y$ and $Z$. This average value, represented by a colored pixel on the maps, is attributed to the bin $i$. 


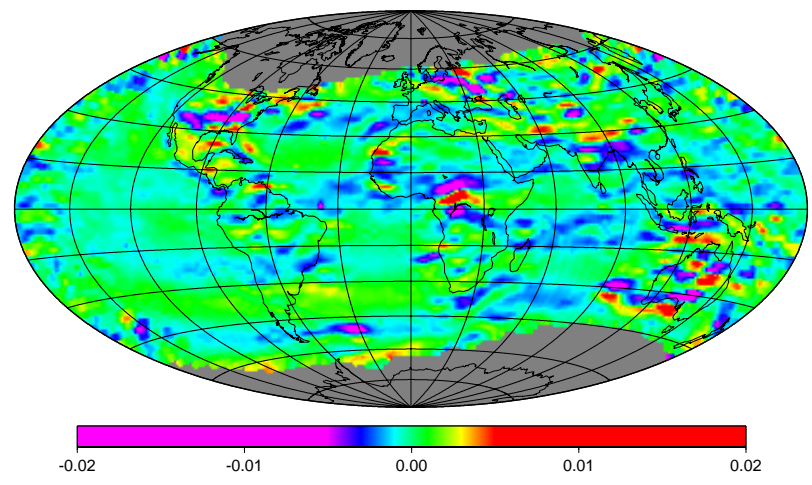

Fig. 2. Along-track second derivative of the crustal field (in $n T / s^{2}$ ) from data in 2001-2004, longitudinal component.

\subsection{Removing the crustal field}

The crustal field has energy in the same spatial frequency domain as the EEJ, so the two fields cannot be separated through their spectral content. However, the crustal field does not change in time, contrary to the EEJ field, which can be assumed to vanish around local time midnight. Therefore, the maps obtained by our analysis at local times around midnight have no contribution from the EEJ, and the same crustal field contribution than at any other local time. We take advantage of this fact and of the adequate accuracy of our maps of averaged quantities, to extract an averaged map computed from 21:00 LT to 03:00 LT, from the other maps, in order to eliminate the crustal field.

\section{Results}

\subsection{Crustal field}

As said earlier, processing the data relative to the night local time - in fact from 21:00 LT to 03:00 LT - provides simply the map of the lithospheric field (Fig. 2). It is, of course, a transformed anomaly map, a map of the along-track second derivative of the intensity anomaly (not the intensity of the anomaly), often considered when dealing with aeromagnetic surveys in which only the intensity is measured. Note that the auroral zones are hidden using an ad hoc mask. The same mask will be used in all the maps presented in the paper.

The map of Fig. 2 is very stable, i.e. is the same when computed from different subsets of the four years of data. It presents the well-known drawbacks and advantages of this kind of map. On the one hand, since the orbits are grossly meridian, the fields of geological structures trending eastwest are amplified and, due to the angle between the main field and the vertical, the anomalies are displaced in the meridian direction with respect to their sources. On the other hand, anomalies can be decoalesced and made easier to interpret. Lithospheric anomaly maps have been derived from
CHAMP and Ørsted data by Maus et al. (2002), following the earlier maps based on MAGSAT data (Cohen and Achache, 1990; Ravat et al., 1995). We will not discuss the lithospheric field in this paper, which is devoted to the equatorial electrojet field.

\subsection{Variations with local time}

In order to study the variations with local time (LT) of the EEJ field, we split the data of the interval 2001-2004 into 24 subsets, corresponding to the following LT intervals: 00:30-01:30, 01:30-02:30, etc., 23:30-00:30. The maps for the longitudinal component at 06:00, 08:00, 10:00, 12:00, 14:00, 16:00 and 18:00 LT are shown in Fig. 3. (Other maps are not shown due to the limited space available.)

The map at 00:00 LT (Fig. 3a) contains no EEJ signal. The remaining features, after removing the crustal field, are indeed very tiny and give an idea of the error in maps at other local times.

A negative signal along the dip equator is visible at 06:00 LT (Fig. 3b). (In fact, it is already slightly visible one hour earlier). This signal is maximal over Africa, where it reaches $-0.005 \mathrm{nT} / \mathrm{s}^{2}$ (i.e. about $-10^{-4} \mathrm{nT} / \mathrm{km}^{2}$ ), but is absent over the Pacific Ocean. It has been previously observed at ground stations in Africa, India and South America and is sometimes referred to as the morning counter electrojet (Gouin and Mayaud, 1967; Mayaud, 1977). The counter electrojet disappears at 08:00 LT everywhere except over South America (Fig. 3c).

Between 10:00 LT and 14:00 LT (Figs. 3d-f), the full EEJ signal is visible. It is made of three parallel bands aligned and symmetrical with respect to the dip equator. Each band has an approximate width of $1000 \mathrm{~km}$. The central band is positive and is caused by the eastward equatorial electrojet. The two flanking bands are negative and of slightly lower intensity; we will show in Sect. 3.5 that they are due to westward return currents. The intensity of the signal is maximal at 12:00 LT. Somewhat unexpectedly, it is slightly higher at 10:00 LT than at 14:00 LT. All three bands have absolute extrema between 0.005 and $0.02 \mathrm{nT} / \mathrm{s}^{2}$ (i.e. $10^{-4}$ and $4 \times 10^{-4} \mathrm{nT} / \mathrm{km}^{2}$ ) at 12:00 LT.

At 16:00 LT (Fig. 3g), the EEJ signal looks like that at 08:00 LT, perhaps with a slightly stronger positive central band. It is maximal over the Eastern Pacific Ocean, a region where it is weak at 08:00 LT.

The dusk features of the EEJ are markedly different from the dawn features. At 18:00 LT (Fig. 3h), the EEJ signal is almost no longer visible. It is the green band along the geomagnetic equator. There is no afternoon counter electrojet.

\subsection{Variations with universal time}

The variations with universal time (UT) are studied in the same manner as the variations with local time, using 24 subsets corresponding to UT intervals 00:30-01:30, 
(a)

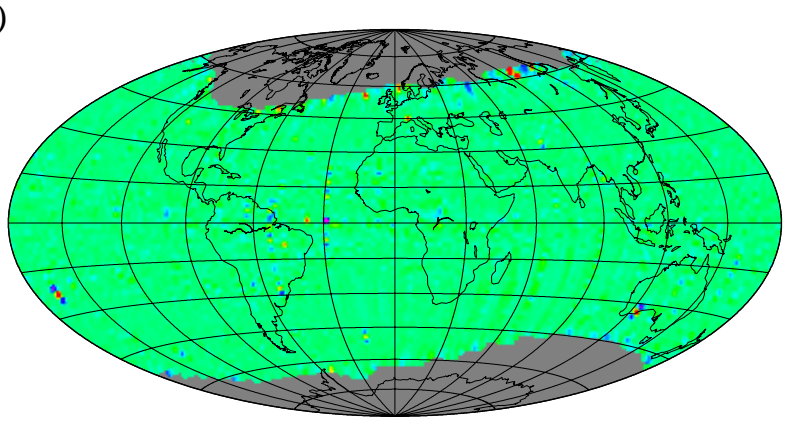

(c)

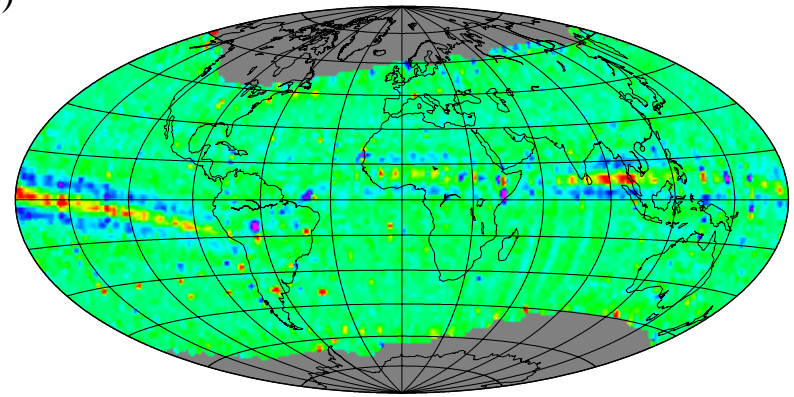

(e)

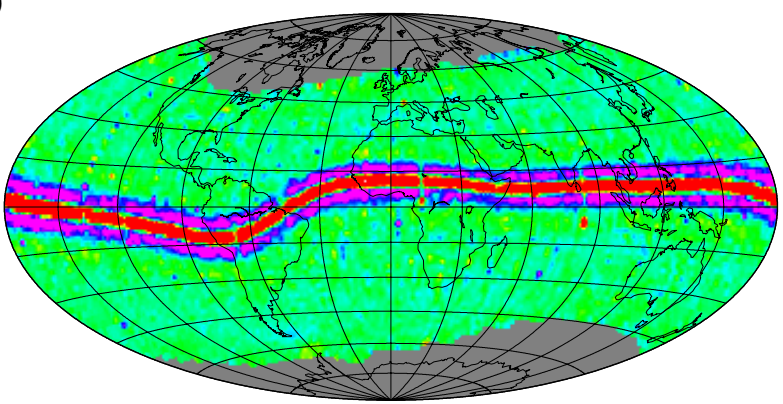

(g)

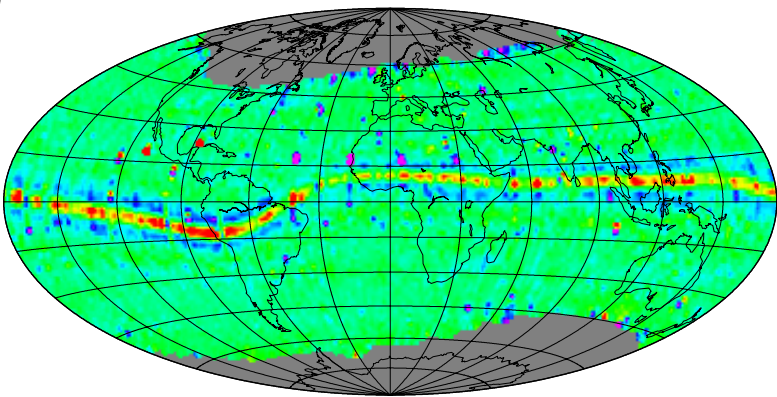

(b)

(d)
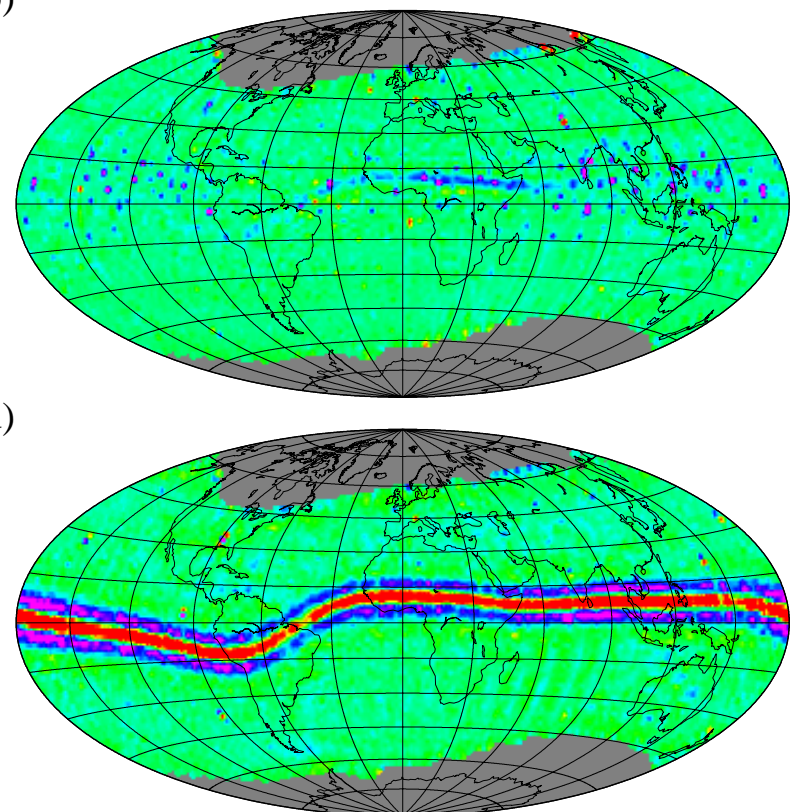

(f)

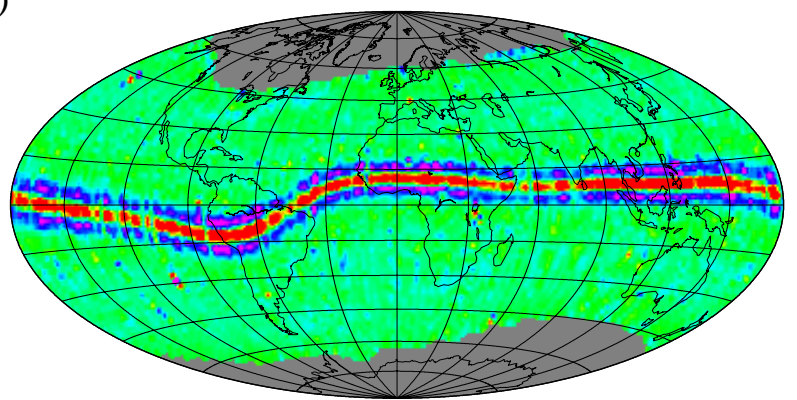

(h)

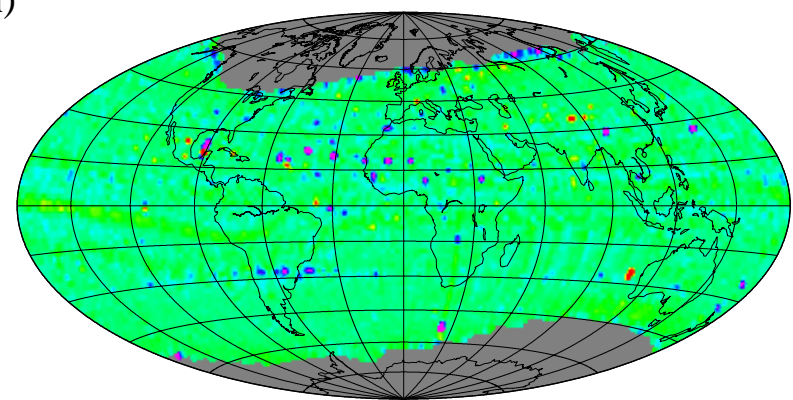

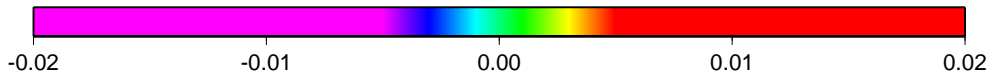

Fig. 3. Variation with local time of the along-track second derivative of the equatorial electrojet field (in $\mathrm{nT} / \mathrm{s}^{2}$ ), longitudinal component, at 00:00 (a), 06:00 (b), 08:00 (c), 10:00 (d), 12:00 (e), 14:00 (f), 16:00 (g) and 18:00 (h).

01:30-02:30, etc., and applying the same analysis to the longitudinal component. The result is an hour-by-hour movie of the EEJ displacement around the world. Expectedly, the EEJ is made of the same three bands as in the LT maps and follows the dip equator. Less expectedly, its length and intensity varies significantly along its path. Due to the limited space available, only the maps at 06:00, 11:00 and 17:00 UT are presented in Fig. 4.

The EEJ at 06:00 UT (Fig. 4a) is made up of three parts: a head at the western side, a body and a tail at the eastern 
(a)

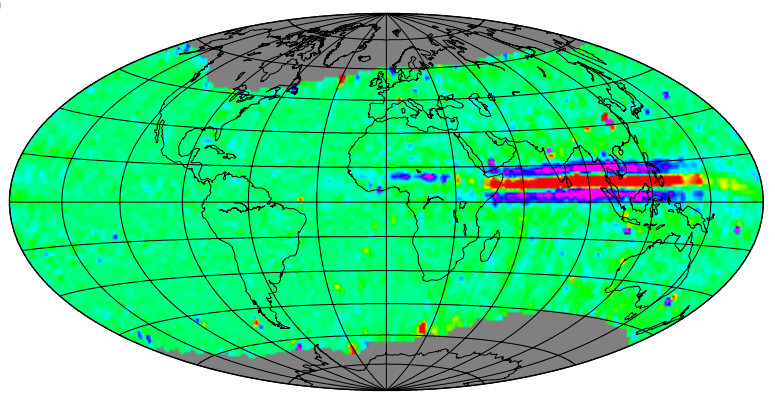

(b)

(c)
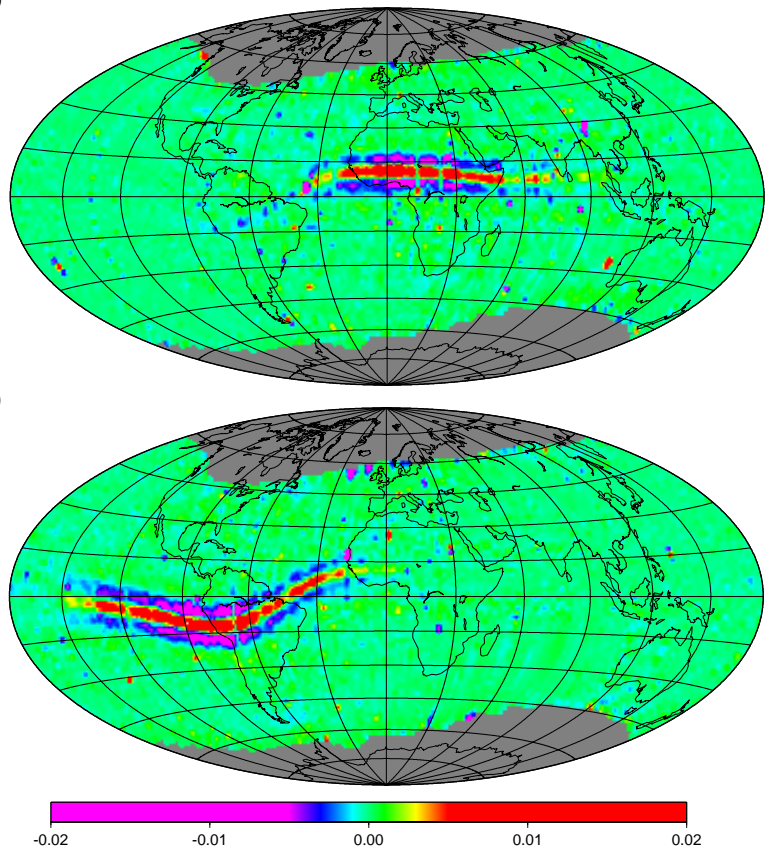

Fig. 4. Variation with universal time of the along-track second derivative of the equatorial electrojet field (in $\mathrm{nT} / \mathrm{s}^{2}$ ), longitudinal component, at 06:00 (a), 11:00 (b) and 17:00 (c).

side. The head and tail consist of several patches within the central band along the dip equator, while the body consists of the three bands visible on LT maps. In good agreement with LT maps, the signal within the head (i.e. at dawn) is negative while the signal within the tail (i.e. at dusk) is positive. The head is significantly longer than the tail. The patches over Africa are located about $2000 \mathrm{~km}$ in front of the EEJ body, while the tail patches are about $1000 \mathrm{~km}$ behind it.

At 11:00 UT (Fig. 4b), the EEJ reaches the point over the Atlantic Ocean where the dip equator bends southward. The body of the EEJ seems to be slightly compressed at its front by this sudden turn. At the same time, both the head and tail expand over several thousands of $\mathrm{km}$ and are longer than at other UT.

At 17:00 UT (Fig. 4c), the opposite situation occurs. The EEJ reaches its maximum length, about $3000 \mathrm{~km}$ from the middle of the Pacific Ocean to the Western coast of Africa. (a)

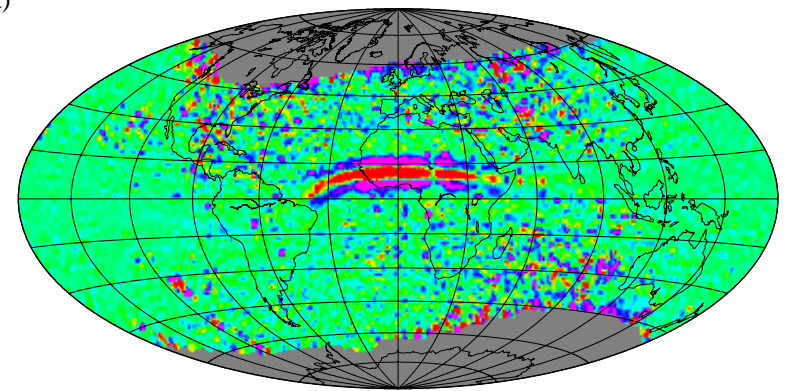

(b)

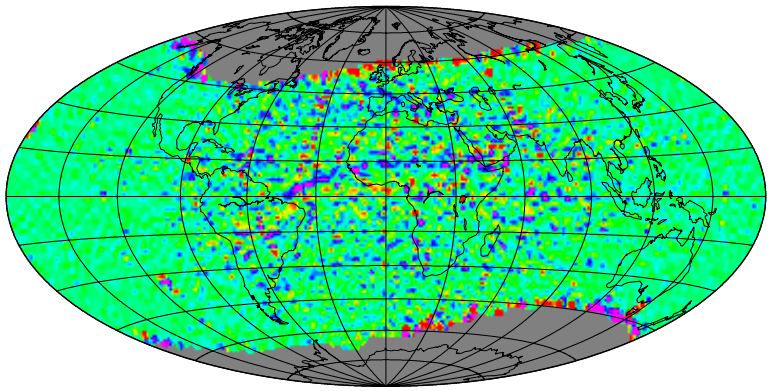

(c)

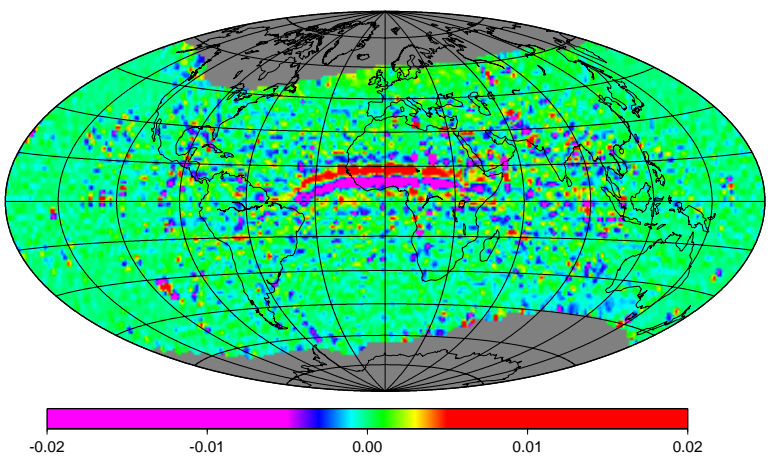

Fig. 5. Along-track second derivative of the equatorial electrojet field (in $\mathrm{nT} / \mathrm{s}^{2}$ ) at 12:00 UT, from the $X(\mathbf{a}), Y(\mathbf{b})$ and $Z$ (c) components.

It is made of entirely of all three band types and has no discernible head or tail.

These variations with UT of the EEJ structure will be further discussed in Sect. 3.6, where the longitude profile averaged over the width of the EEJ will be quantified and plotted for several UT.

\subsection{Cartesian components}

Figure 5 shows maps obtained from the along-track second derivatives of the $X, Y$ and $Z$ components of the field, at 12:00 UT. The EEJ is conspicuous on both the $X$ and $Z$ maps, much less on the $Y$ map.

The $X$ map looks very much the same as the corresponding map for the longitudinal component, which is not surprising, as the horizontal field direction is nearly aligned with the horizontal north direction, except in the region over South America, where the dip equator is curved. The main difference 


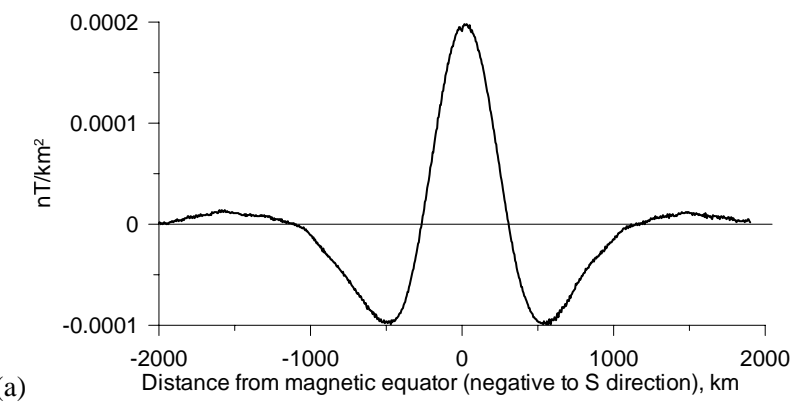

(a)

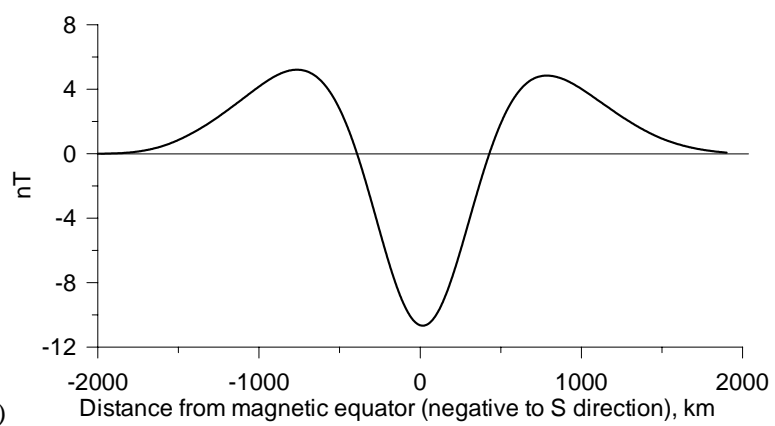

(b)

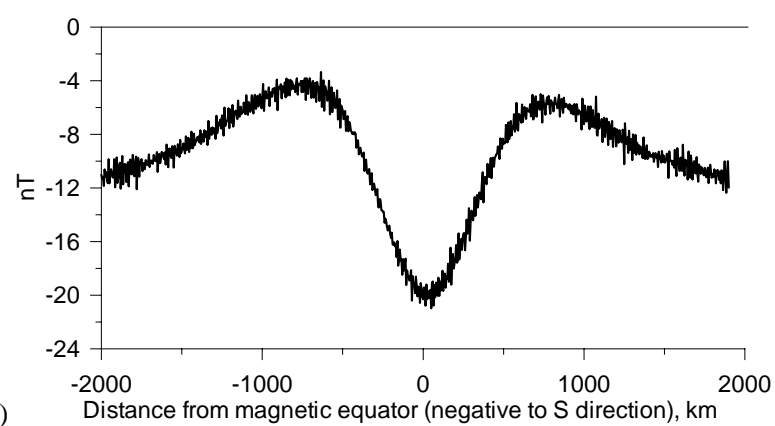

Fig. 6. Profile normal to the dip equator of the equatorial electrojet field, longitudinal component, using a step of $5 \mathrm{~km}$ : along-track second derivative, in $\mathrm{nT} / \mathrm{km}^{2}$ (a); twicely integrated along-track second derivative, in nT (b); original signal, before treatment, in nT (c).

between Fig. 5a and Fig. 4 is the amount of small-scale features at mid-latitudes. These features are widely spread and mainly concentrated around 06:00 LT and 18:00 LT. They are roughly symmetrical with respect to the dip equator.

The $Y$ component map shows the same kind of features at mid-latitudes, although not concentrated at the same local times. They are almost uniformly distributed between 06:00 LT and 18:00 LT. The EEJ signal is weak and is made of two bands roughly parallel to the dip equator, of opposite signs. It is negative north of the equator and positive south of the equator. These features could be due to meridional currents flowing northward and southward from the EEJ.

Small-scale features are also present in the $Z$ component map. They are distributed at lower latitudes than for the two other components and are concentrated around 06:00 LT and 18:00 LT. The EEJ signal is as strong as for the $X$ component

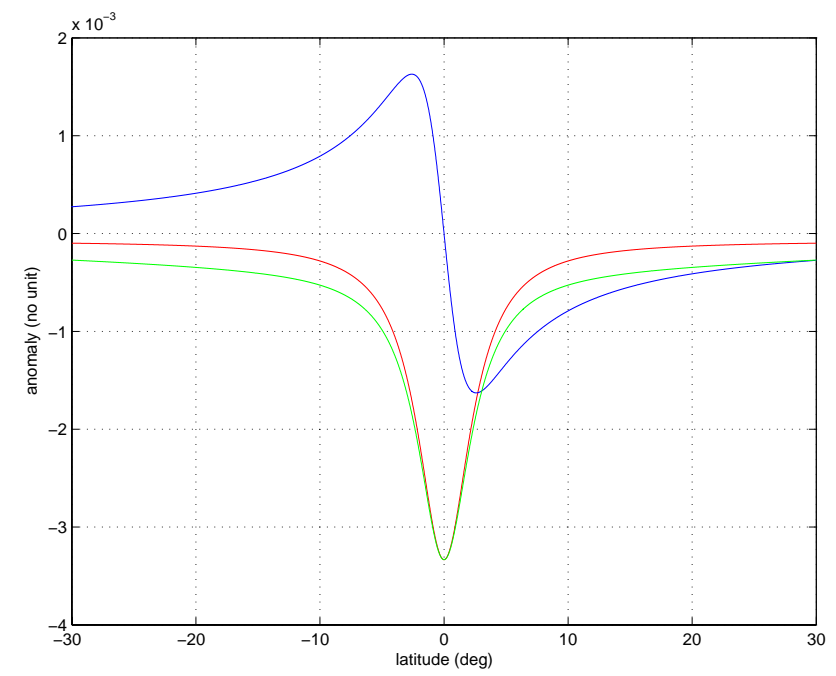

Fig. 7. Profile normal to the dip equator of the magnetic anomaly at the satellite altitude generated by a single current line along the equator when the main magnetic field is assumed axial dipolar: $X$ component (red curve), $Z$ component (blue curve) and $F$ component (green curve).

but has a different shape. There are two extrema, antisymmetrical about the dip equator, which is in good agreement with theoretical predictions for a linear current flowing eastward like the EEJ.

The distribution of the mid-latitude small-scale features suggest that they are due to mid-latitude field-aligned currents (Olsen, 1997) (indeed these features do not appear on the longitudinal component maps). Such currents electrically connect the two hemispheres during the day. Due to boundary effects, the magnetic field they generate is mainly northward or southward at dawn and dusk. Also, the vertical component is larger near the equator where the magnetic field lines become horizontal (see Sect. 4).

\subsection{Profile normal to the dip equator}

The profile normal to the dip equator of the EEJ field, longitudinal component, is represented in Fig. 6a, using a step of $5 \mathrm{~km}$. It is averaged along the whole equator. The main positive peak is centered exactly on the equator and reaches $2 \times 10^{-4} \mathrm{nT} / \mathrm{km}^{2}$, i.e. about $0.01 \mathrm{nT} / \mathrm{s}^{2}$. The secondary negative peaks are about $500 \mathrm{~km}$ apart and reach $10^{-4} \mathrm{nT} / \mathrm{km}^{2}$, i.e. about $0.005 \mathrm{nT} / \mathrm{s}^{2}$. This is in good agreement with previous maps (Figs. 3 and 4 ).

The same profile, twice integrated in the direction perpendicular to the dip equator (using the same step of $5 \mathrm{~km}$ ), is shown in Fig. 6b. It can be checked in Fig. 6c that the averaged profile is similar to the original profile before treatment. Expectedly, the along-track second derivative has filtered out the large-scale variation associated with the $S_{q}$ current system, without attenuating the EEJ signal. (High-frequency 


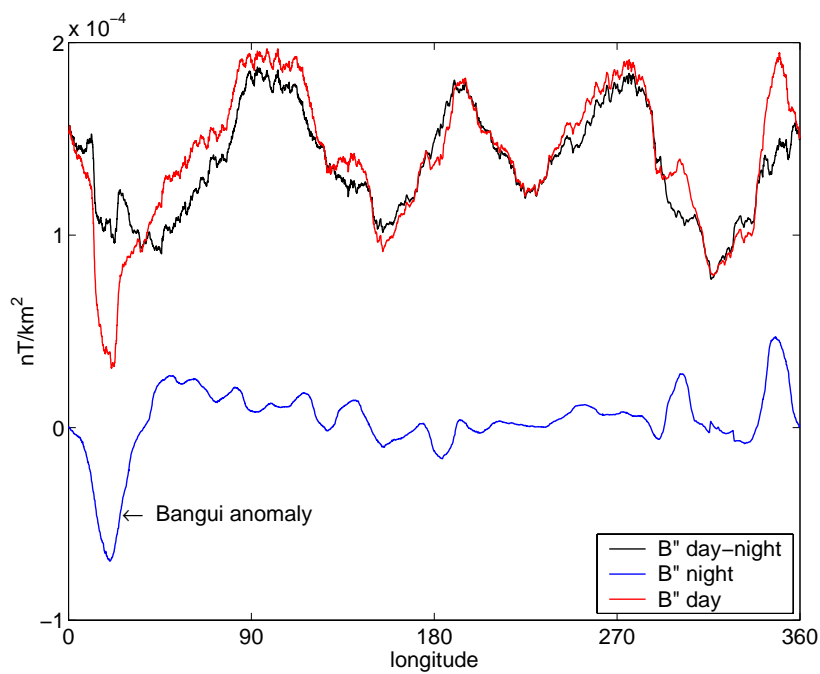

Fig. 8. Profile along the dip equator of the equatorial electrojet field, longitudinal component (in $\mathrm{nT} / \mathrm{km}^{2}$ ), using data from the whole interval 2001-2004 (in black); results obtained without removing the crustal field, using data from $6 \mathrm{~h}$ of day only (in red) and $6 \mathrm{~h}$ of night only (in blue). The anomaly is projected onto the normal to the dip equator and then averaged over a 500-km wide and 1300-km long window sliding along the equator.

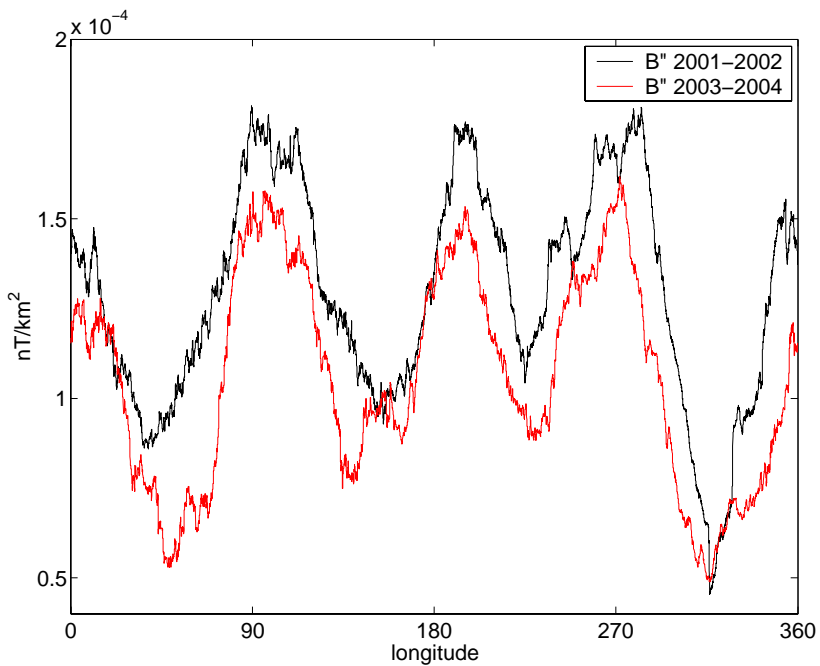

Fig. 9. Comparison of the longitude profiles of the equatorial electrojet field, longitudinal component (in $\mathrm{nT} / \mathrm{km}^{2}$ ), obtained from 2001-2002 data only (in black) and 2003-2004 data only (in red).

noise is present in Fig. $6 \mathrm{c}$ and not in Fig. 6a because the data are averaged over all longitudes without being averaged in $2.5^{\circ} \times 2.5^{\circ}$ bins, as this is the case when producing Figs. $6 \mathrm{a}$ and $6 \mathrm{~b}$.)

Figures $6 \mathrm{~b}$ and $\mathrm{c}$ show that the main peak in the second derivative curve corresponds exactly to the central peak of the raw field anomaly. The secondary peaks in the second derivative curve are, as expected, slightly shifted from their

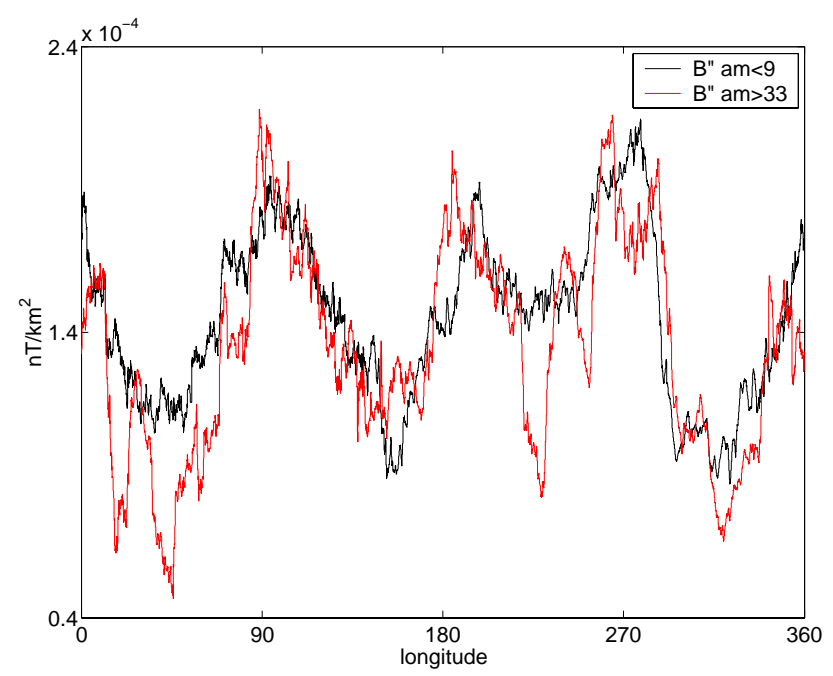

Fig. 10. Variation with geomagnetic activity of the longitude profile of the equatorial electrojet field, longitudinal component, in $\mathrm{nT} / \mathrm{km}^{2}: a m<9$ (in black), $a m>33$ (in red).

positions in the raw field curve, by about $250 \mathrm{~km}$ towards the dip equator. However, the ratio of intensities between these peaks and the main peak, about 2 , is almost not affected by the second derivative.

While the central band of the EEJ signal on the maps in Figs. 3 and 4 is clearly caused by an eastward electric current along the dip equator, the origin of the two flanking bands, visible from 08:00 LT to 16:00 LT, is less obvious. They could be caused by the eastward band of the current itself or by westward return currents on each side of the central eastward current. To shed light on this issue, let us assume that the main field is axial dipolar (hence the dip equator and geographic equator are the same) and consider a single eastward current line along the equator at the altitude $100 \mathrm{~km}$. Line current models are widely used in the literature (e.g. Fambitakoye and Mayaud, 1976), although they are often more complicated than this one. The field anomaly generated by this current line at the altitude $400 \mathrm{~km}$ is calculated in Appendix $\mathrm{A}$ and is represented as a function of the latitude in Fig. 7. There is no flanking high on the $X$ and $F$ components, because the profile is calculated well above the electric current line and the spherical geometry of the Earth is taken into account. (The same calculation applied to a zero altitude indeed leads to flanking highs.) The comparison of Figs. 6b and 7 suggests that the two flanking bands require westward return currents on each side of the central EEJ.

\subsection{Profile along the dip equator}

The longitude profile of the EEJ field, longitudinal component, is calculated using a $500-\mathrm{km}$ wide and $1300-\mathrm{km}$ long window sliding along the dip equator; $500 \mathrm{~km}$ is roughly the width of the uppermost part of the EEJ peak (see Fig. 6a), and 
(a)

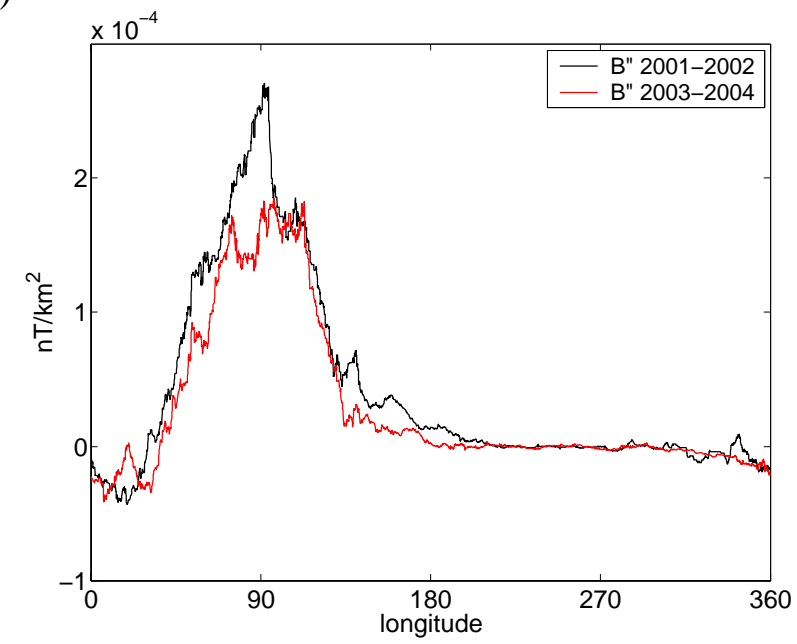

(b)

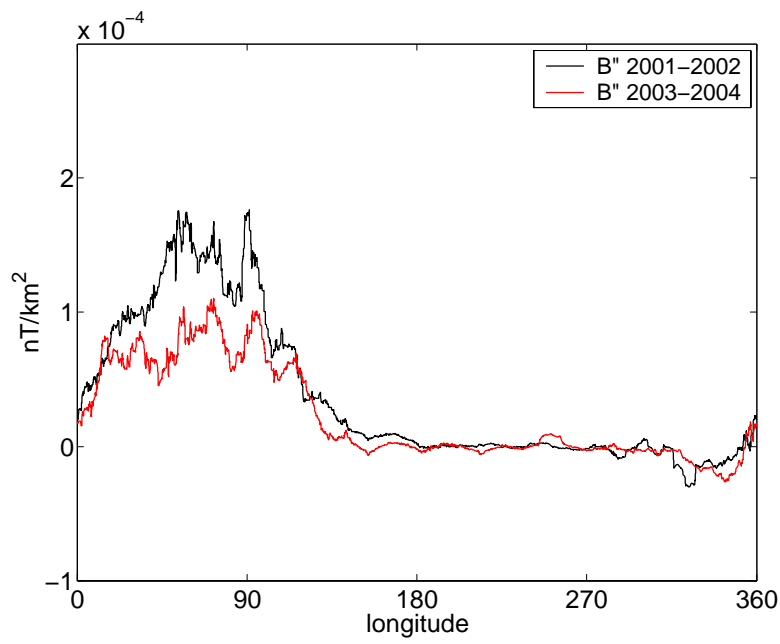

(c)

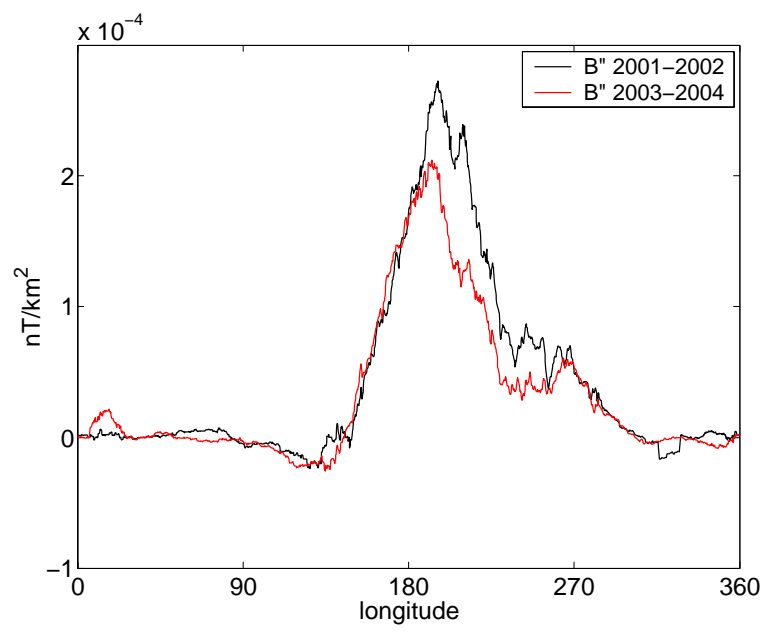

Fig. 11. Variation with universal time of the longitude profile of the equatorial electrojet field, longitudinal component, in $\mathrm{nT} / \mathrm{km}^{2}$. The profiles are computed over 2001-2002 (black curves) and 2003-2004 (red curves) at 06:00 (a), 08:00 (b) and 22:00 (c).

$1300 \mathrm{~km}$ has been chosen in order to average out the fluctuations associated with the satellite altitude variations.

The resulting curve for 2001-2004 is displayed in Fig. 8, as well as the two curves obtained without removing the crustal field, by selecting data from $6 \mathrm{~h}$ of night only and $6 \mathrm{~h}$ of day only. The night curve displays short scale features due to the crustal field, in particular a large negative peak in Africa associated with the Bangui anomaly. No fluctuation due to the satellite altitude variations is visible. As expected, the difference between the night and day curves is close to the EEJ longitude profile, which displays four main peaks almost equally distributed around the dip equator, at around $0^{\circ}$, $90^{\circ}, 180^{\circ}$, and $270^{\circ}$ longitude. The field second derivative in these peaks is roughly twice that outside the peaks.
As shown in Fig. 9, the shape of the longitude profile is almost the same in 2001-2002 and 2003-2004. As 2001-2002 and 2003-2004 are two distinct data sets, we can conclude that the four-peak structure is very robust. The major difference between the two curves is that the 2003-2004 one is slightly but systematically lower than the 2001-2002 one. In other words, the EEJ intensity varies with solar activity, as solar activity regularly decreases from 2001 to 2004 . This is not surprising as the ionospheric conductivity is strongly dependent upon the UV radiation flux, which is governed by solar activity. This effect is very significant, as it overcomes the increase in the signal caused by the regular altitude decrease of CHAMP (from $450 \mathrm{~km}$ in 2001 down to $370 \mathrm{~km}$ at the end of 2004). 
(a)

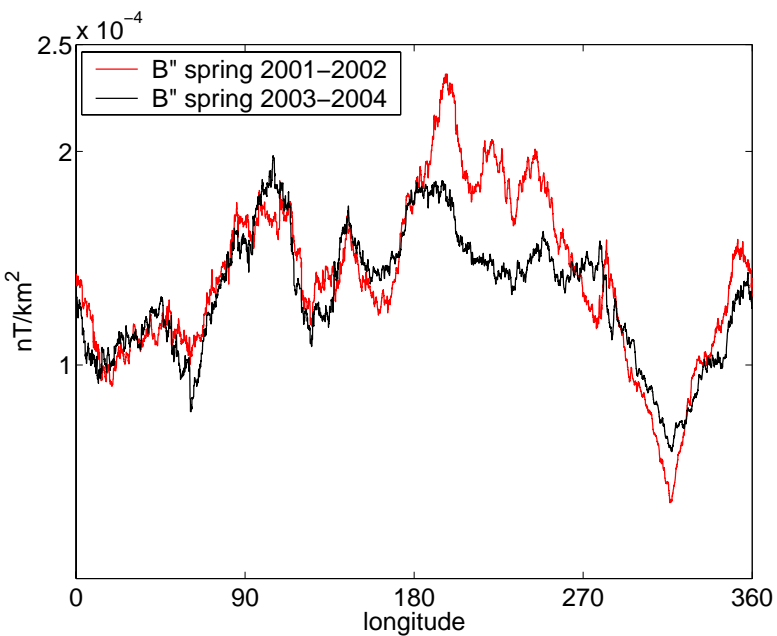

(c)

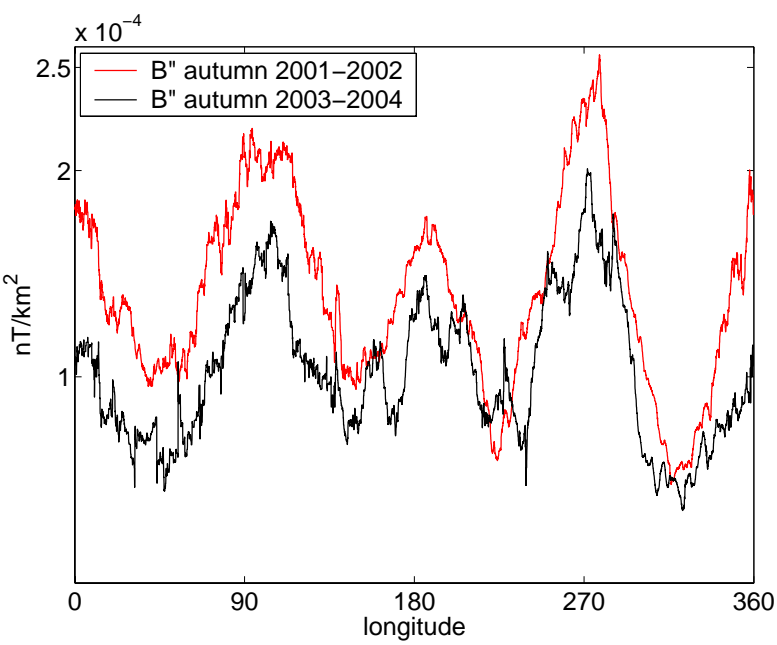

(b)

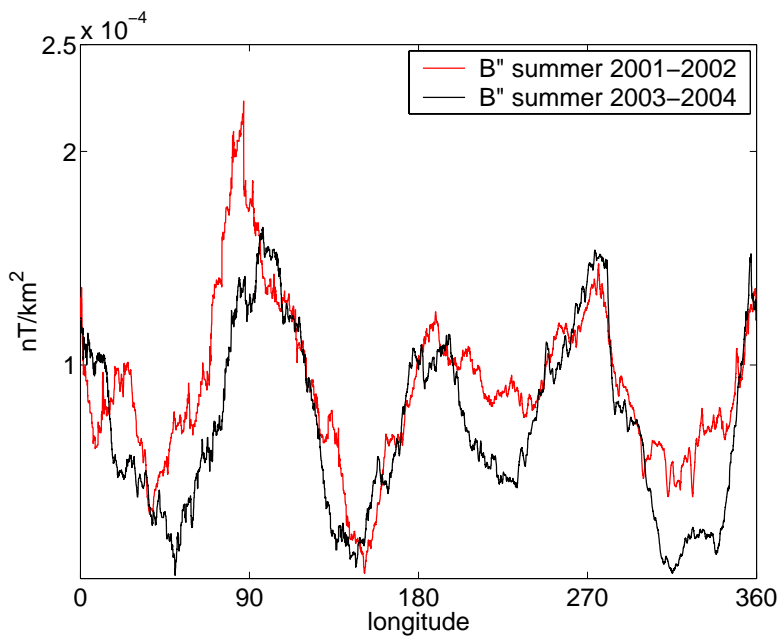

(d)

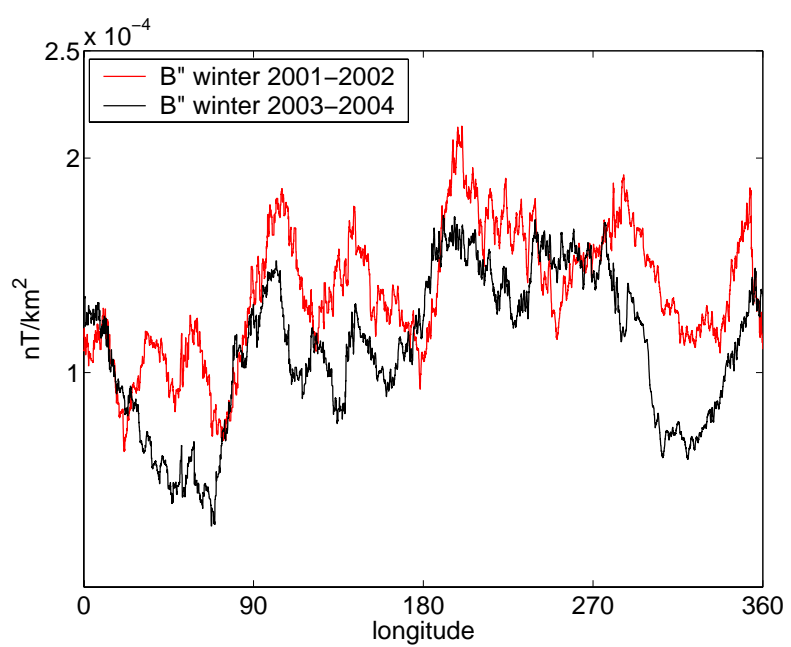

Fig. 12. Seasonal variation of the longitude profile of the equatorial electrojet field, longitudinal component, in $\mathrm{nT} / \mathrm{km}^{2}$ : spring (a), summer (b), autumn (c) and winter (d). The profiles are computed over 2001-2002 (red curves) and 2003-2004 (black curves).

In order to check whether geomagnetic activity also has an influence on the EEJ intensity, we have analysed the variation with geomagnetic activity of the EEJ longitude profile using the $a m$ index to select data. We chose am instead of $K p$ because am relies on a large network of observatories and is directly expressed in nT (Mayaud, 1980). The obtained curves are displayed in Fig. 10. Somewhat surprisingly, there is no clear effect of geomagnetic activity on the EEJ intensity. The anomaly on quiet days is alternately larger and smaller along the dip equator than the anomaly on disturbed days.

Figure 11 shows the variation with universal time of the longitude profile of the EEJ field for the time intervals 20012002 and 2003-2004. At 06:00 UT (Fig. 10a), the longwavelength features are the same for both time intervals, although short-wavelength features are somewhat different. As noted previously, the 2001-2002 anomaly is slightly more intense than the 2003-2004 one. On both curves, the counter electrojet is clearly visible at the left side of the peak, in good agreement with the anomaly map displayed in Fig. 4a. At 08:00 UT, the peak is much smaller; this corresponds to a low in the averaged longitude profile (see Fig. 8). Again, the large-scale features of the profile are very stable from one time interval to the other. At 22:00 UT, the EEJ is slightly in advance in 2003-2004 with respect to 2001-2002, but the tail is less intense. All these snapshots show that the main features of the EEJ longitude profile are very robust over four years. 
The seasonal variation of the EEJ longitude profile is shown in Fig. 12, using data from the time intervals 20012002 and 2003-2004. The profiles for 2001-2002 look very similar to those for 2003-2004, which suggests that the observed seasonal variability is a robust feature, stable over at least four consecutive years. There is a strong variability of the EEJ intensity from one season to the other. The EEJ anomaly is maximal in spring and autumn and minimal in summer. The difference between summer and winter mainly comes from lower lows in summer, rather than from higher peaks in winter. The four peaks are clearly visible in summer and autumn, less visible in spring and winter. (Note that splitting the data decreases the number of data per bin.)

\section{Discussion}

The method of analysis used in the present paper resorts to no modeling, except that of the main field (which could, in principle, be skipped), no a priori assumption regarding the structure of the EEJ, nor any other current system, and no complicated filtering. It has been checked that each step of the analysis removes the signal generated by one or several well-identified sources.

- The efficiency of the along-track second derivative for removing large-scale fields of external and internal origin has been optimized by fine-tuning down to $20 \mathrm{~s}$ the step used when computing the derivative in Eq. (2).

- It has been shown that the projection onto the main field direction removes almost all of the field-aligned current effect. This is illustrated in Fig. 5, where the alongtrack second derivatives of the anomaly Cartesian components (and not that of the longitudinal projection) are shown.

- The main features of the anomaly map obtained from nightside data are in good visual agreement with crustal field maps obtained by other methods. For example, we also find that bigger anomalies are systematically located on continents.

- The averaging over enough long periods of time significantly smooths the day-to-day variability, which is high for magnetic fields produced by ionospheric sources; the results obtained from various subsets of data are indeed consistent.

These precautions make the obtained maps and profiles very robust and very little room is left for the unwanted signal in them.

The maps in local time and universal time presented in Figs. 3 and 4 are actually parts of two 24-picture movies. More pictures in each movie could be produced by the same method, if needed. These movies are, to our knowledge, the first global visualizations of the EEJ anomaly at all local and universal times. For example, Ivers et al. (2003) studied only a few local times, as made available by the slowly drifting Ørsted satellite, while Lühr et al. (2004) only focussed on the EEJ at noon local time.

The structure of the EEJ field, as revealed by the movies, confirms the global existence of phenomena observed previously in a few places. In particular, we find that the counterelectrojet is present in the morning only, as observed by Fambitakoye and Mayaud (1976) from ground stations in Central Africa, and later by Cohen and Achache (1990) from Magsat dusk and dawn data. The cause of this phenomenon is still unknown; further theoretical investigations are now needed to make progress on this issue.

Westward return currents are clearly visible on the maps, starting as soon as the main eastward electrojet in the morning and vanishing simultaneously with it at dusk. Their inferred size at noon is in good agreement with that obtained by Lühr et al. (2004). Meridional currents are also visible in the $Y$ component, as previously detected by Olsen (1997) and Sabaka et al. (2004). The closure of the electric circuit is still an open question. Unfortunately, our approach does not make it straightforward to calculate an electric intensity budget, so that we are unable, at the present time, to directly compare with previously published budgets.

The determination of the longitude profile of the EEJ field is only possible thanks to the global coverage provided by satellite data. This is the reason why this issue has arisen only recently in the literature and is still debated, although some preliminary work has been done by Ravat and Hinze (1993) using Magsat data. Our results are in good agreement with those of Jadhav et al. (2002b) and Ivers et al. (2003) from Ørsted data, who also observe four quasi-regularly spaced peaks along the dip equator. There is less agreement with the longitude profile presented by Lühr et al. (2004) (see their Fig. 8), which only has two peaks. The difference could come from the various models introduced by Lühr et al. (2004) to extract the EEJ from the data: spherical harmonics models of the core field, the crustal field and the $S_{q}$ current system, and a priori model of the EEJ current density.

\section{Conclusions}

In the present paper we have analysed the EEJ from CHAMP satellite data, using a new method based on the computation of the along-track second derivative. This method is a simple and efficient way to extract the EEJ signal from satellite data by filtering out contributions from the other sources and without resorting to any modeling, except that of the main field. It has been applied to the full CHAMP data set over four years, thus enabling one to study variations of the EEJ with local time, universal time, solar activity, geomagnetic activity and seasons. The main conclusions of this analysis are: 


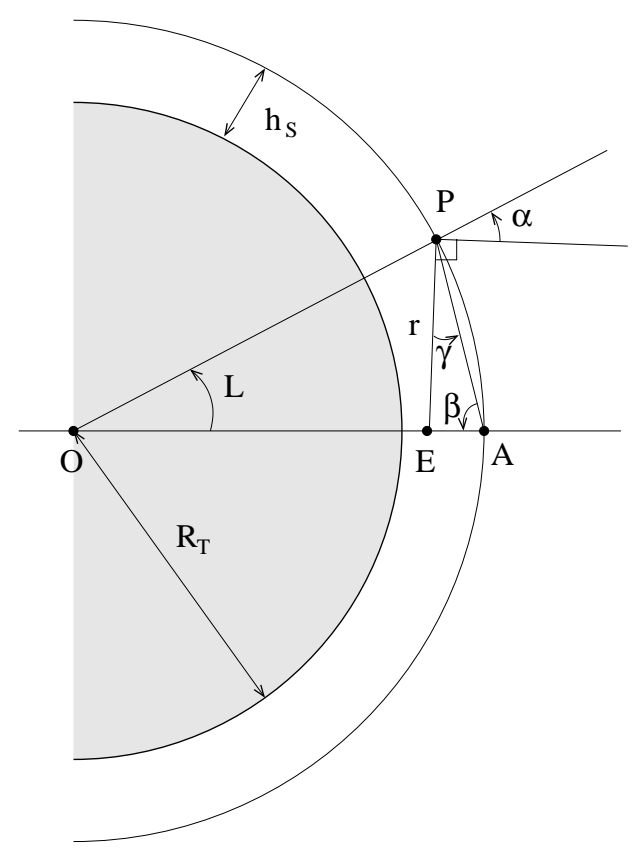

Fig. A1. Geometric notations used in the derivation of the field anomaly generated by a single current line along the equator when the main magnetic field is assumed axial dipolar.

1. The electrojet is made up of a central band of current flowing eastward and two lateral, less intense, bands of current flowing westward; the total size of the EEJ is about 2000-km width.

2. There exists a morning counter electrojet over a large fraction of the dip equator; there is no afternoon counter electrojet.

3. The EEJ length varies along its path over the dip equator; it is compressed when the equator bends southward.

4. Meridional EEJ currents are visible on the $Y$ component.

5. Small-scale features, which could be attributed to interhemispheric currents, are visible on the $X, Y$ and $Z$ components at mid-latitudes.

6. The EEJ longitude profile displays four regularly spaced peaks.

7. The EEJ intensity decreases with solar activity, which suggests that it is strongly dependent upon the UV radiation flux.

8. The EEJ does not vary with geomagnetic activity.

9. The EEJ is minimum in summer and maximum in spring and fall.
In a further study, we will compare our results with those predicted by the comprehensive model CM4 of Sabaka et al. (2004).

\section{Appendix A}

\section{Field generated by a current line at satellite altitude}

Let us consider a single eastward current line along the geographic equator at an altitude $h_{E}=100 \mathrm{~km}$ and assume the main geomagnetic field is axial dipolar. The purpose of this Appendix is to obtain the expression of the field anomaly generated by this current line along the orbit of a CHAMPlike satellite, assumed to be polar and circular, at an altitude $h_{S}=400 \mathrm{~km}$. This is a 2-D problem, to be solved in the meridian plane; see Fig. A1.

The north and downward vertical components of the anomaly at a point $P$ on the orbit may be expressed as

$$
\begin{aligned}
& \Delta X=-\frac{\mu_{0} i}{2 \pi r} \sin \alpha, \\
& \Delta Z=-\frac{\mu_{0} i}{2 \pi r} \cos \alpha,
\end{aligned}
$$

where $i$ is the current intensity, $r$ the distance from the current line and $\alpha$ the angle between the anomaly field and the vertical direction at $P$. Then the scalar anomaly is

$\Delta F \approx-\frac{\mu_{0} i}{2 \pi r}(\sin \alpha \cos I+\cos \alpha \sin I)$,

where $I$ is the local inclination of the main field.

In the case of an axial dipolar magnetic field, it is wellknown that $I$ may be related to the latitude $L$ using

$I=\arctan (2 \tan L)$.

Using classical trigonometric formulae, we find that

$\alpha=\frac{\pi}{2}-\beta+\gamma$,

$\beta=\frac{\pi-L}{2}$,

$\gamma=\arctan \left[\frac{\Delta h \sin \beta}{2\left(R_{T}+h_{S}\right) \sin \left(\frac{L}{2}\right)-\Delta h \cos \beta}\right]$,

where $R_{T}$ is the Earth's radius, $\Delta h=h_{E}-h_{S}$ and $\beta$ and $\gamma$ are both defined as in Fig. A1. Therefore, the angle $\alpha$ is given by

$\alpha=\frac{L}{2}-\arctan \left[\frac{\Delta h \operatorname{cotan}\left(\frac{L}{2}\right)}{2\left(R_{T}+h_{S}\right)-\Delta h}\right]$.

Substituting Eqs. (A4) and (A8) into Eq. (A3), we may calculate the profile of the anomaly due to the current line along the orbit of the satellite. 
Acknowledgements. We thank T. Sabaka for his thorough reading of the manuscript and for his helpful suggestions. We thank S. Byrdina for helping with the calculations. We thank P.-N. Mayaud for many interesting discussions. We also thank the two referees for helpful comments. This is IPGP contribution No. 2114.

Topical Editor M. Pinnock thanks D. Ravat and another referee for their help in evaluating this paper.

\section{References}

Cohen, Y.: Traitements et interprétations de données spatiales en géomagnétisme: étude des variations latérales d'aimantation de la lithosphère terrestre, Ph.D. thesis, Université Paris VII, 1989.

Cohen, Y. and Achache, J.: New global vector magnetic anomaly maps derived from Magsat data, J. Geophys. Res., 95, $10783-$ $10800,1990$.

Doumouya, V., Vassal, J., Cohen, Y., Fambitakoye, O., and Menvielle, M.: Equatorial electrojet at African longitudes: first results from magnetic measurements, Ann. Geophys., 16, 658676, 1998,

\section{SRef-ID: 1432-0576/ag/1998-16-658.}

Egedal, J.: The magnetic diurnal variation of the horizontal force near the magnetic equator, Terr. Magn. Atmos. Electr., 52, 449451, 1947.

Fambitakoye, O. and Mayaud, P. N.: Equatorial electrojet and regular daily variation $S_{R}$; II, the center of the equatorial electrojet, J. Atmos. Terr. Phys., 38, 19-26, 1976.

Forbes, J. M.: The Equatorial Electrojet, Rev. Geophys. Space Phys., 19, 469-504, 1981.

Forbush, S. E. and Casaverde, M.: Equatorial Electrojet in Peru, Tech. Rep. 620, Carnegie Inst. Wash., Washington, D.C., 1961.

Gouin, P. and Mayaud, P. N.: A propos de l'existence possible d'un "contre électrojet" aux latitudes magnétiques équatoriales, Ann. Geophys., 23, 41-47, 1967.

Ivers, D., Stening, R., Turner, J., and Winch, D.: Equatorial electrojet from Ørsted scalar magnetic field observations, J. Geophys. Res., 108(A2), 1061, doi:10.1029/2002JA009310, 2003.

Jadhav, G., Rajaram, M., and Rajaram, R.: Main field control of the equatorial electrojet: a preliminary study from the Oersted data, J. Geodyn., 33, 157-171, 2002a.

Jadhav, G., Rajaram, M., and Rajaram, R.: A detailed study of equatorial electrojet phenomenon using Ørsted satellite observations, J. Geophys. Res., 107(A8), 1175, doi:10.1029/2001JA000183, $2002 b$.
Langel, R. A., Purucker, M., and Rajaram, M.: The equatorial electrojet and associated currents as seen in Magsat data, J. Atmos. Terr. Phys., 55, 1233-1269, 1993.

Langlais, B., Mandea, M., and Ultré-Guérard, P.: High-resolution magnetic field modeling: application to MAGSAT and Ørsted data, Phys. Earth Planet. Inter., 135, 77-91, 2003.

Lühr, H., Maus, S., and Rother, M.: Noon-time equatorial electrojet: Its spatial features as determined by the CHAMP satellite, J. Geophys. Res., 109, A01 306, doi:10.1029/2002JA009656, 2004.

Maus, S., Rother, M. amd Holme, R., Lühr, H., Olsen, N., and Haak, V.: First scalar magnetic anomaly map from CHAMP satellite data indicates weak lithospheric field, Geophys. Res. Lett., 29(14), doi:10.1029/2001GL013685, 2002.

Mayaud, P. N.: The equatorial counter electrojet - A review of its geomagnetic aspects, J. Atmos. Terr. Phys., 39, 1055-1070, 1977.

Mayaud, P. N.: Derivation, Meaning and Use of Geomagnetic Indices, Geophysical Monograph 22, Am. Geophys. Union, Washington, D.C., 1980.

Olsen, N.: Ionospheric $F$ region currents at middle and low latitudes estimated from Magsat data, J. Geophys. Res., 102(A3), 45634576, 1997.

Olsen, N.: Swarm End-to-End Mission Performance Simulator Study, Tech. rep., Danish Space Research Institute, ESA contract No. 17263/03/NL/CB, 2004.

Rastogi, R. G.: The Equatorial Electrojet: Magnetic and Ionospheric Effects, in Geomagnetism, edited by: Jacobs,J. A., vol. 3, chap. 7, Academic Press, London, 461-525, 1989.

Ravat, D. and Hinze, W. J.: Considerations of variations in ionospheric field effects in mapping equatorial lithospheric Magsat magnetic anomalies, Geophys. J. Int., 113, 387-398, 1993.

Ravat, D., Langel, R. A., Purucker, M., Arkani-Hamed, J., and Alsdorf, D. E.: Global vector and scalar Magsat magnetic anomaly maps, J. Geophys. Res., 100(B10), 20 111-20 136, 1995.

Rigoti, A., Chamalaun, F. H., Trivedi, N. B., and Padilha, A. L.: Characteristics of the equatorial electrojet determined from an array of magnetometers in N-NE Brazil, Earth Planets Space, 51, 115-128, 1999.

Sabaka, T. J., Olsen, N., and Langel, R. A.: A comprehensive model of the quiet-time, near-Earth magnetic field: phase 3, Geophys. J. Int., 151, 32-68, 2002.

Sabaka, T. J., Olsen, N., and Purucker, M.: Extending comprehensive models of the Earth's magnetic field with Ørsted and CHAMP data, Geophys. J. Int., 159, 521-547, 2004. 